\title{
Alignment of Red-Sequence Cluster Dwarf Galaxies: From the Frontier Fields to the Local Universe
}

\author{
Wayne A. Barkhouse ${ }^{1}$, Haylee Archer ${ }^{1}$, Jaford Burgad ${ }^{1}$, Gregory \\ Foote $^{1}$, Cody Rude ${ }^{1}$ and Omar López-Cruz ${ }^{2}$ \\ ${ }^{1}$ Department of Physics and Astrophysics, University of North Dakota, \\ Grand Forks, North Dakota, USA \\ email: wayne. barkhouse@und.edu \\ ${ }^{2}$ Instituto Nacional de Astrofísica, Optica y Electrónica \\ Tonantzintla, Mexico \\ email: omarlx@inaoep.mx
}

\begin{abstract}
We investigate the alignment effect of red-sequence dwarf galaxies using a sample of 121 low-redshift galaxy clusters and cluster galaxies selected from the HST Frontier Fields.
\end{abstract}

Keywords. galaxies: clusters: general - galaxies: structure

\section{Introduction}

Galaxy clusters are the largest virialized structures in the universe. Due to their high density and mass, they are an excellent laboratory for studying the environmental effects on galaxy evolution. Numerical simulations have predicted that tidal torques acting on dwarf galaxies as they fall into the cluster environment will cause the major axis of the galaxies to align with their radial position vector (a line that extends from the cluster center to the galaxy's center). We have undertaken a study to measure the redshift evolution of the alignment of red-sequence cluster dwarf galaxies based on a sample of 57 low-redshift Abell cluster imaged at KPNO using the 0.9-meter telescope, and 64 clusters from the WINGS dataset (Fasano et al. 2006). To supplement our low-redshift sample, we have included galaxies selected from the Hubble Space Telescope Frontier Fields. Leveraging the HST data allows us to look for evolutionary changes in the alignment of red-sequence cluster dwarf galaxies over a redshift range of $0<z<0.35$. The alignment of the major axis of the dwarf galaxies is measured by fitting a Sersic function to each red-sequence galaxy using GALFIT (Peng et al. 2002). The quality of each model was checked visually after subtracting the model from the galaxy. The cluster sample was then combined by scaling each cluster by $r_{200}$. No statistically significant evidence was found for an alignment of red-sequence dwarf galaxies with: 1) the major axis of the brightest cluster galaxy, 2) the major axis of the cluster defined by the position of cluster members, and 3) a radius vector pointing from the cluster center to individual galaxies. Our cluster sample was sub-divided into different radial regions and redshift bins.

\section{References}

Fasano, G., Marmo, C., Varela, J., D'Onofrio, M., Poggianti, B. M., Moles, M., Pignatelli, E., Bettoni, D., Kjaergaard, P., Rizzi, L., Couch, W. J., \& Dressler, A. 2006, A\&3A, 445, 805 Peng, C. Y., Ho, L. C., Impey, C. D., \& Rix, H.-W. 2002, AJ, 124, 266 